\title{
Diabetes mellitus, fever, proptosis, and rapid loss of vision
}

\author{
Ravinder Goswami, Nikhil Tandon, Sanjay Thulkar, Narayana Kochupillai
}

A 12-year-old boy was referred to the hospital with a history of diabetes mellitus diagnosed 20 days earlier following an episode of ketoacidosis. During this episode he developed fever, a bloodstained discharge from the right nostril, proptosis, redness and rapid deterioration of vision in the right eye culminating in blindness within 10 days. Examination revealed a sick, conscious, febrile child with weight of $25 \mathrm{~kg}$. There was mucopurulent discharge from the right eye, complete external ophthalmoplegia with absence of light perception and direct light reflex. Investigations revealed total leukocyte counts of $12 \times 10^{9} / 1$ with $64 \%$ polymorphs and $30 \%$ lymphocytes, blood glucose of $18 \mathrm{mmol} / \mathrm{l}$ and traces of ketones in the urine. Purulent discharge from the eye was sent for culture and diagnostic external ethmoidectomy from the right eye was undertaken. Computed tomography (CT) of the orbit (figure 1) and histopathological specimen findings (figure 2) are given below. Glycaemic control was achieved with multiple injections of crystalline bovine insulin before each major meal. The first-line drug for treating his orbital condition was not available in the hospital stock. Pending availability, oral ketoconazole was given at a dose of $200 \mathrm{mg}$ twice daily for two weeks.

\section{All India Institute of Medical Sciences, New Delhi 110029, India \\ Department of Endocrinology, \\ R Goswami \\ $\mathrm{N}$ Tandon \\ N Kochupillai \\ Department of \\ Radiodiagnosis \\ $S$ Thulkar \\ Correspondence to Dr N Kochupillai}

Accepted 22 November 1995

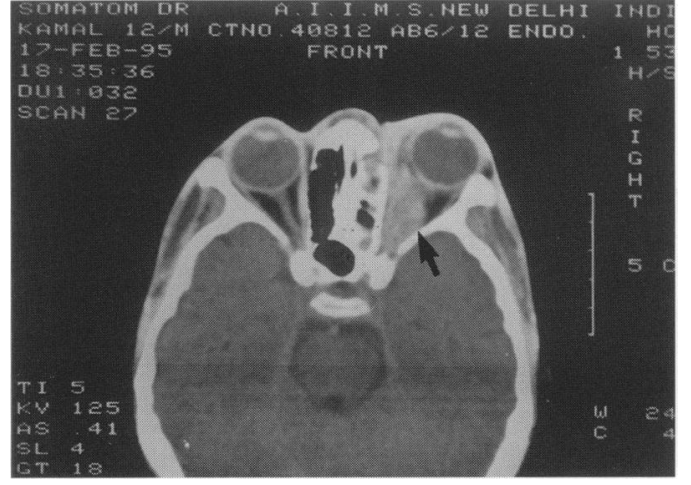

Figure 1 CT scan through the orbit

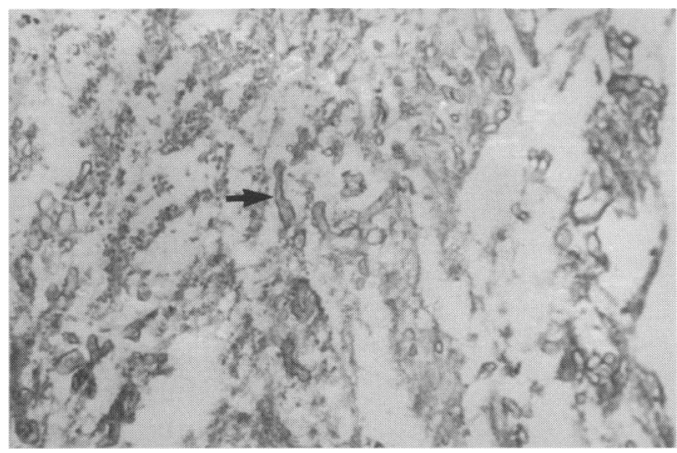

Figure 2 Light microscopic picture of histopathological specimen from the external ethmoidectomy 


\begin{tabular}{|l|}
\hline Predisposing \\
conditions for \\
mucormycosis \\
\hline - diabetes mellitus \\
- haematological \\
malignancy \\
- burns \\
- AIDS \\
- malnutrition \\
- post organ \\
transplant \\
- renal failure \\
- intravenous drug \\
abuse \\
- amoebiasis \\
- kwashiorkar \\
\hline
\end{tabular}

Box 1

\section{Answers}

\section{QUESTION 1}

Figure 1 is a contrast-enhanced CT scan of the orbit revealing an ill-defined soft tissue mass with optic nerve entrapment in the right orbit partially obliterating the retrobulbar fat planes. In addition, right maxillary sinus reveals mucosal thickening. There is no evidence of bony destruction or invasion of intracranial tissue by the soft tissue mass.

\section{QUESTION 2}

Figure 2 is the histopathology specimen revealing broad nonseptate fungal hyphae with nonparallel margins suggestive of mucormycosis.

\section{QUESTION 3}

In the setting of diabetes mellitus with poor glycaemic control and ketosis, painful proptosis, redness and blood-stained nasal discharge are strong indicators of fungal infection due to mucormycosis. Presence of soft tissue mass in the orbit and demonstration of nonseptate hyphae on histopathological examination further suggest the clinical diagnosis of orbital mucormycosis. In fact, fungal culture of the purulent discharge confirmed the growth of Zygomycetes in this patient.

\section{QUESTION 4}

Amphotericin B, since its introduction in 1957, is the standard treatment of diabetic mucormycosis. Its use has considerably reduced the mortality from $90 \%$ in the untreated state to $40 \% .^{1}$ However, occurrence of sideeffects with amphotericin B has led some workers to use alternative forms of antifungal therapy. Ketoconazole was approved for treatment of fungal infection in 1981. Since then, five reports are described in the literature where ketoconazole has been used to treat diabetic mucormycosis. In two reports, this drug was used in combination with amphotericin B and therefore, no conclusions could be drawn on the efficiency of ketoconazole. ${ }^{2,3}$ In another report of cutaneous mucormycosis in a diabetic woman, trial of ketoconazole was without success and patient was later successfully treated with amphotericin B. ${ }^{4}$ The patient described by Kumar et $a \bar{P}$ was treated with itraconazole. There has been only one case to date where ketoconazole has been effective in the treatment of diabetic mucormycosis. ${ }^{6}$

In the present case, during the two weeks of treatment with ketoconazole, there was objective evidence of deterioration in the form of worsening redness of the eye, mucopurulent discharge and significant increase in the orbital soft tissue mass. In view of the clinical deterioration, ketoconazole was discontinued and therapy with amphotericin B commenced $(1 \mathrm{mg} / \mathrm{kg} /$ day $)$. A total dose of $1.5 \mathrm{~g}$ was given over a period of eight weeks. On amphotericin B treatment, progressive improvement in ocular movement and reduction in soft tissue mass was noticed. At the end of therapy there was no proptosis and full eyeball movements were regained. However, vision did not improve and fundus examination showed optic atrophy. No side-effect were noticed except for fever, chills and rigors during the infusion of amphotericin.

\section{Discussion}

\section{PREDISPOSING CONDITIONS FOR}

\section{MUCORMYCOSIS}

Mucormycosis is an opportunistic infection found in debilitated hosts (box 1). It occurs in $c$ several forms, including orbital, rhino-orbital, $\vec{F}$ rhino-orbito-cerebral, pulmonary, gastrointestinal, cutaneous and disseminated forms. Among the various clinical forms of mucormycosis, rhino-orbito-cerebral and cutaneous disease are more frequently noticed in patients with diabetes mellitus.

\section{DIABETES MELLITUS AND MUCORMYCOSIS}

Diabetes mellitus is the most common underlying disease associated with mucormycosis; about $76 \%$ of mucormycosis infections are 3 associated with diabetes mellitus. This affinity for diabetic patients is related to the fact that $N$ this fungus thrives in an environment rich in $\mathrm{N}$ glucose and acid pH. Diabetic patients with of poor glycaemic control also have decreased $\omega$ phagocytic activity of polymorphonuclear leu- 을 kocytes. Susceptibility is further increased in ketoacidosis because of the attendant increase in serum free iron levels. This facilitates the growth of rhizopus, which is an iron-requiring organism (box 2).

CLINICAL FEATURES

Symptoms seen in patients with mucormycosis are listed in box 3. Presenting signs of hemiplegia, facial necrosis and nasal deformity carry a poor prognosis. ${ }^{1}$

\section{DIAGNOSTIC INVESTIGATIONS}

White cell counts are increased in these patients and may be used to monitor the response to treatment. A biopsy should be

\begin{tabular}{|l|}
\hline Causes of increased susceptibility to \\
mucormycosis in diabetes mellitus \\
\hline - increased serum iron levels in diabetic \\
ketoacidosis \\
- enhanced fungus survival in acid- and glucose- \\
rich environment \\
decreased phagocytic activity of neutrophils in \\
ketoacidosis \\
\hline
\end{tabular}

Box 2

\begin{tabular}{|c|}
\hline $\begin{array}{l}\text { Clinical features of diabetic } \\
\text { mucormycosis }\end{array}$ \\
\hline $\begin{array}{l}\text { - headache }(73 \%) \\
\text { - fever ( } 55 \%) \\
\text { - black nasal eschar (45\%) } \\
\text { orbito-fascio-cellulitis (36\%) } \\
\text { - cranial nerve palsy }(36 \%) \\
\text { - altered sensorium }(30 \%) \\
\text { - hemiparesis }(27 \%)\end{array}$ \\
\hline
\end{tabular}

Box 3 
taken to look for broad, irregular nonseptate hyphae. Fungus can be cultured on Sabouraud medium. The most frequent finding on routine radiographs is haziness of the paranasal sinuses. Soft tissue invasion, necrosis and early bone erosion are better demonstrated by computed tomography. Magnetic resonance imaging, with or without gadolinium, is the best way of evaluating intracranial extension including carotid artery thrombosis and cavernous sinus thrombosis. Serology has no place in the diagnosis of mucormycosis. ${ }^{1}$

\section{TREATMENT}

Therapeutic options for mycormycosis in a diabetic patient are listed in box 4 . The serious nature of mucormycosis necessitates rapid infusion of effective doses of amphotericin B, rather than gradual incremental dose. The usual dose is $0.5-1 \mathrm{mg} / \mathrm{kg} /$ day dissolved in $5 \%$ dextrose solution. Because of the fungistatic rather than fungicidal nature of the drug, treatment is given for a prolonged period. A cumulative dose of $1.5-4 \mathrm{~g}$ is given and cessation of treatment is guided by clinical signs and symptoms, including reduction of fever, wound healing, negative cultures, and decreasing white cell count.

In spite of the considerable success which has been achieved with amphotericin B in treating mucormycosis, toxic effects have been the limiting factors. These include nephrotoxicity, bone marrow depression, cardiotoxicity and hypokalaemic weakness. Recently, the use of liposomal amphotericin has shown considerable improvement in the treatment of mucormycosis. The drug can be used in much higher doses without any significant risk of the abovementioned side-effects. In fact, side-effects such as drug-infusion-induced chills, rigors, and fever are also rare with this formulation.

Amphotericin has also been used in conjunction with rifampicin and interferon alpha-2 in a few cases. However, because of scanty data availability, no conclusion can be drawn at this stage. $^{1}$

1 Blitzer A, Lawson W. Fungal infections of the nose and paranasal sinuses. Otolaryngol Clin North Am 1993; 26: paranasal

2 O'Keefe M, Haining WM, Young JD, Guthrie W. Orbital mucormycosis with survival. $\mathrm{Br} f$ Ophthalmol 1986; 70: $634-6$

3 Reich H, Behr W, Barnert J. Rhinocerebral mucormycosis in a diabetic ketoacidotic patient. $\mathcal{F}$ Neurol 1985; 232: $115-$ 7.

4 Eschard JP, Poynard JP, Janody D, et al. Cutaneous mucormycosis in a diabetic woman. Diagnostics and therapeutic problems. Rev Med Interne 1984; 5: 298-302.

\section{Therapeutic considerations in} treatment of diabetic mucormycosis

- glycaemic control

- amphotericin B (liposomal modification)

- azole group of antifungals not recommended

- surgical debridement

- hyperbaric oxygen

\section{Box 4}

Surgery has an important role in the management of mucormycosis. In early cases without necrosis, simple nasal, transantral or ethmoidal biopsy and curettage should be performed. Patients with extensive necrosis require more radical debridement. A blind eye should not be removed as a routine because there are no data showing that the survival rate is enhanced after orbital exenteration. Hyperbaric oxygen is reported to inhibit the growth of Zygomycetes in vitro, and has been tried in a few patients with rhinocerebral mucormycosis. ${ }^{1}$

\section{PROGNOSIS}

The factors related to lower survival rate in patients with mucormycosis include delayed diagnosis and treatment, hemiparesis or hemiplegia, bilateral sinus involvement, leukaemia, renal disease, treatment with deferoxamine, facial necrosis, and nasal deformity. Age and sex of the patients are not important determinants of survival. ${ }^{1}$ Orbital mucormycosis has a more indolent course and, with adequate treatment, mortality is reduced to $40 \%$.

\section{Final diagnosis}

Orbital mucormycosis in a diabetic patient.

Keywords: diabetes mellitus, mucormycosis, amphotericin $\mathrm{B}$, ketoconazole

5 Kumar B, Kaur I, Chakarbarti A, Sharma VK. Treatment of deep mycosis with itraconazole. Mycopathologica 1991; 115: $169-74$

6 Barnert J, Behr W, Reich H. An amphotericin B-resistant case of rhinocerebral mucormycosis. Infection 1985; 13: $134-6$ 